\title{
A Novel Approach for Selection of Appropriate Software as a Service in Cloud Computing
}

\author{
Mehul Mahrishi \\ Central University of Rajasthan
}

\begin{abstract}
Cloud Computing is an awesome technology. It has to goahead and annexes the computing world. The development of cloud computing embed its sprouting continually in the recent era. Cloud Computing have contrived clan very lavish comfortable to perform their chore. As it's fundamental definition says that As You Pay As You Go. In this delving work we talk about the cloud standards and some standard parameters to enhance the cloud request scheduling. We have used rough set theory to generate the mathematical model. The algorithm deals with the scheduling of the requests on the basis of some parameters that we have identified to achieve the best optimal paths or cloud service provider allotment to the users. The algorithm is implemented in the cloud simulator CLOUDSIM in which cloudlets, datacenters, cloud brokers are created to perform the algorithms. Finally, we created a GUI for the user convenience so that both Cloud Service Provider and users can themselves analyze each others performance. We have reused some inbuilt packages of CLOUDSIM net beans to simulate the process.
\end{abstract}

\section{Keywords}

Cloud Computing $\square \square$ Cloud Service Providers $\square \square$ Rough Set Theory, Datacenters, Users, Parameters, CLOUDSIM

\section{INTRODUCTION}

In the day today world Computing is moving in its seven havens where it is making its place like a worm in a fruit. The most important thing to see here is its play a vital in businesses. Number of people wants to save their money, time, space and improve performance of there day to day IT activities. This increment done is due to the people requirements in the web data. Advent of Internet plays a very vital role in developing concept of running the Wireless Mobile Phones, Internet Connectivity by Dongle or other wireless Devices, T.V, Radio etc. So from all over analysis the basic definition of the cloud computing can be given as the A style of computing where massively scalable (and elastic) IT- related capabilities are provided as a service to external customers using Internet technologies. A paradigm of computing which tells about both the applications providing services with the help of internet and avail- able scalable hardware and the software running on the systems that may provide the services.

A cloud service provider is responsible for delivering the user demands as a service, which results in cost reduction and efficientlyamalgamation, which treat with ability, security and reliability. Some major cloud service providers are: Ad host Internet, Blue Fire, Cloud more etc. Every CSP (Cloud Service Providers) has their own mechanism of providing services. For example Adhost provides dedicated web hosting featuring Microsoft servers, including windows 2008 and IIS whereas Enter host has their expertise in disaster recover solutions,
Fig. 1 Basic Structure of Cloud Computing

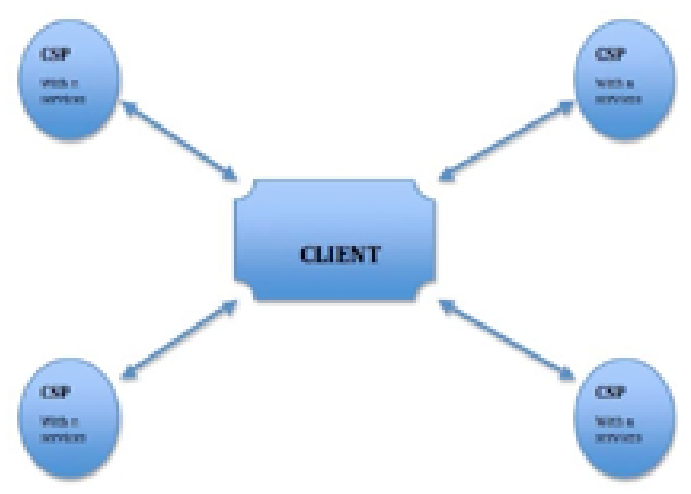

redundant storage and backup services as shown in figure 1[3] [5]. The study of the artifacts provided by various cloud standard-generating organizations, which are providing the various certification and protocols shows that each incoming request can be categorized into one of the 12 standard parameters.

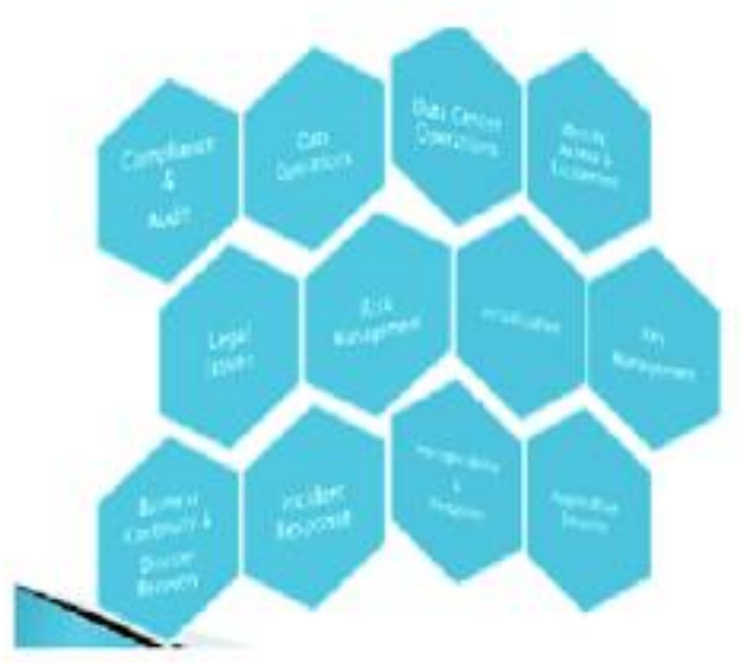

Fig 2. Top Parameters for incoming request to CSP

\section{PROBLEM STATEMENT}

There is a need to standardize cloud. The technology is growing just like Internet use to grow in its time. It can be thought that some of the standards may be too early or too late and some may prove to be inadequate, duplicative, or inappropriate. 
There will be bumps on the roads, but cloud computing will be the major computing paradigm, and the development of standards will play a key role in facilitating the development of the marketplace.

The method of selecting a Cloud Service Provider is evaluated on the basis of Which-Cloud Provider-Provide What.

\section{RELATED WORK}

Few research works focus on Resource Management ofcloud requests already exist. These researches mainly focuses on the type of incoming requests. The user has to enter the type of request and on the basis of some algorithmic parameters like priority, reliability etc. the resource it allocated. The parameters also included are Security and Performance, Resource management, high Sys- tem Performance, Quality of Services and Service level agreements measures. One of the research works uses the matchmaking algorithm

for scheduling of requests. In this algorithm order of incoming request is based on two parameters, Advance Reservation Request (AR) and On Demand Request (OD). The main drawback of this algorithm was found that it does not support co- scheduling of resources and handling uncertainty.

Another paper that we have sited is A Pragmatic Scheduling for Optimal priority. This research work reflects the handling technical aspects of tasks. The tasks are divided into Business Requirements and Technical Requirements.

Here the coordinated scheduling is made for achieving both the requirements. The methodology used in the paper is mathematical formulas, which calculate queues with a function made with both $\mathrm{Tp}$ and $\mathrm{Bp}$. Then it calculates the Poisson distribution rate on the basis of tasks. At Last author had calculated the total resultant priority based on demand divided incoming tasks. The conclusion that was found at the last is priority of each job that arrives and hybridization of $\mathrm{Tp}$ and Bp.

The Third paper we have selected is Ant Colony Optimization Scheduling Algorithm. The problem found under this research work are related to the quality of service requirements such as storage, calculations and evaluations of the cloud services, services with high re- liability and low cost etc.. The model, which is used for the scheduling of cloud, may include the cloud service agency, analysis module, Scheduling module, Service level arrangement monitoring module, Cloud Service Repository and many more modules in the algorithms. The formulation uses DAG for minimization and scheduling algorithms based on ACS (Ant Colony System algorithm). At last author gets to know about the use of SLA monitoring module to monitor the running condition of cloud services.
Another research includes Cost Efficient Scheduling Algorithms. The problem found under this are to maximize the utilization of the internal infrastructure and minimize the cost of running tasks and Computational and data transfer costs. The procedures used by finding one by one steps that are: Application memory Request, Application Deadline factor, Application Data set size, Tasks per application, task run time, Application Memory Request etc. At last the author had concluded the Cost efficient scheduling, Linear Programming Formulation and Public and Hybrid Scheduling Algorithms.

\section{TECHNIQUES USED}

We have seen that many researchers are working in the same direction but with different approaches. The goal is same i.e. to improve and optimize the service provider scheduling [2] [3]. The algorithms for task ordering on the basis of their arrival and task mapping are already proposed [2] [4].

We are proposing a similar kind of approach but to a level above that of job scheduling. It is a responsibility of the cloud computing service provider to provide the adequate service level satisfaction, so we are proposing to device an algorithm which made possible the cloud middleware to determine capability of CSP by using Rough Set analysis on the basis of level of satisfaction of service.

Rough set model can be handled with objects and its characteristics. Here we are considering service providers as objects and its characteristics based on some defined standard parameters.

We have gone through number of artifacts and resource documents to search and summarize 12 major standard parameters. For the sake of simplicity and understanding of the algorithm we are just using 5 parameters out of 12 .

Data Operation, which deals with the different data operation such as searching of data either on the basis of content or location. Moreover some security and access mechanism issues are also discussed. Lastly, it also touches the data persistence methods

Risk Management, which we may deal about Organizations shall develop and maintain a cloud oriented risk management framework to manage risk as defined in the master agreement or industry best practices and standards.

Legal Issues, which we have found that it deals with the legal issues that could be generated when data is moved to the cloud, issues regarding NDA and other agreements between CSP and the customer and other issues that are resolved under laws and litigations of a country.

Compliance And Audit, which Customer and provider both must understand the importance and should follow the implications on existing compliances, audits and other best practices. 
Inter-portability and Portability, explained to interoperability features provided should support security of the data exchanges and messages at protocol level, policy level and identity level. There shall be a mechanism to support integration of services, each of which has a different security mechanism.

\section{PROBLEM FORMULATION}

In our previous research paper we have generated the ROSP Algorithm. The main aim of the algorithm is to find the optimal fuzzy value for each cloud service provider and allot the tenant the CSP with the maxi- mum fuzzy value. In this research paper we have enhanced our algorithm, removed some bugs and even provide a GUI environment so that the users feel it easy to operate.

As per our Algorithm, there are number of Cloud Service Providers which may contain the number of Datacenters.

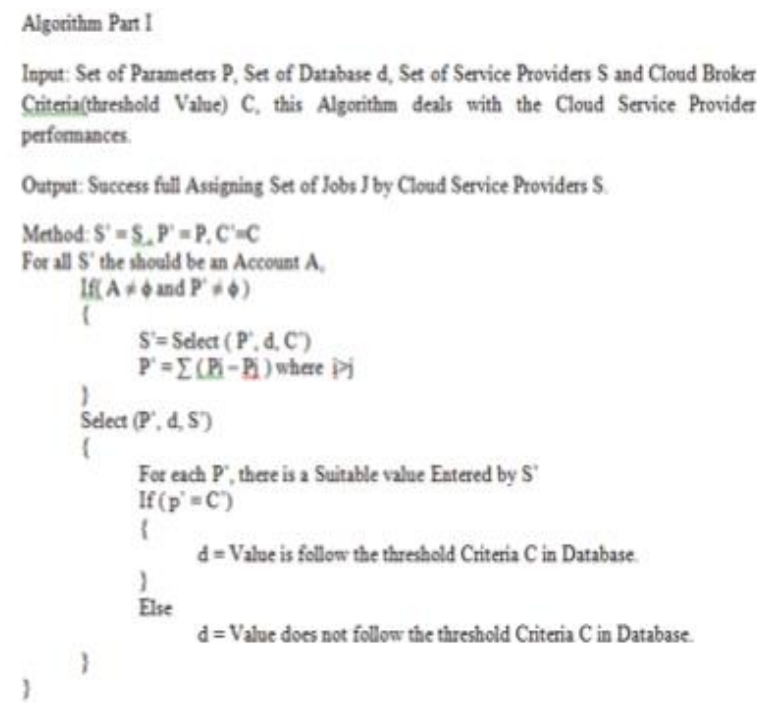

The Part 1 of the algorithm elaborates how we are extracting the relevance values for Cloud Service Providers.

For each CSP, whenever it logs in to the middleware, it needs to create an account, which includes answering of certain relevant questions based on our identified parameters as shown in the figure: 7 .

Each question has some evaluation criterion, through which we calculate the average relevance factor, which is placed in the table shown in figure 8 .

As soon as the table is filled, the ROSP algorithm will execute at the CSP side and extract the optimal CSP on the basis of achieved fuzzy values.

The Part 2 of the algorithm executes at the client side.

After logged in and availing the services of cloud service provider, the tenant needs to answer some questions as similar to service provider. The feedback given by the tenant will be incorporated over the existing value of the CSP in table 1. Therefore we named it as User Adaptive Approach.

\section{RESULTS \& SIMULATION}

As proceeding with our previous ROSP Algorithm, in our Simulation we have used some existing packages of
CloudSim to create Cloud Service Providers, Datacenter, Network etc. In addition to this we have created some packages in NetBeans.

The following results shown are on the simulator CloudSim

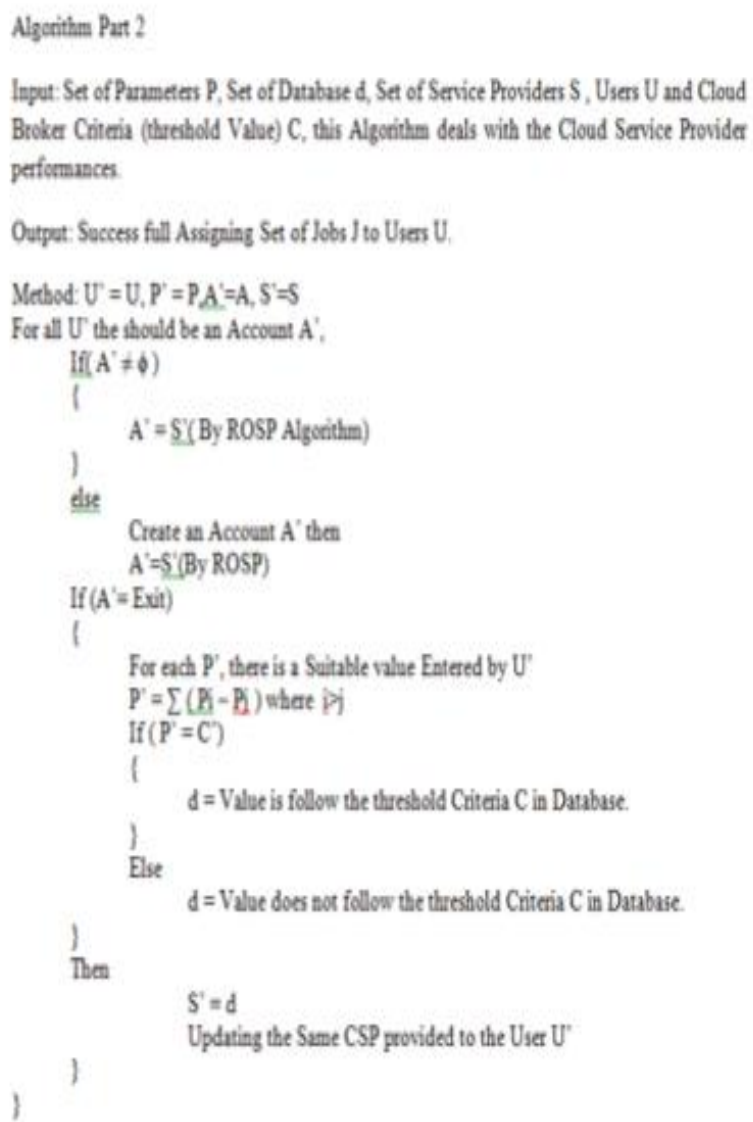

whereas using Netbeans creates the GUI. Moreover, some packages from CloudSim are exported to Netbeans to create

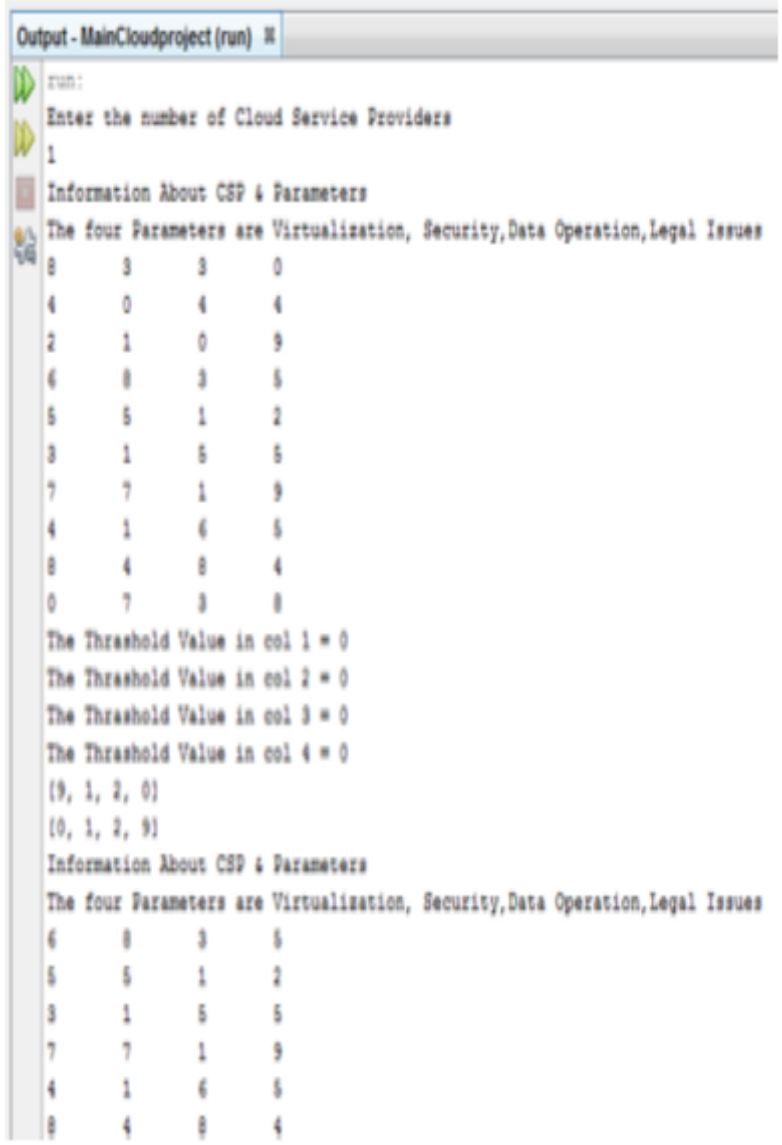



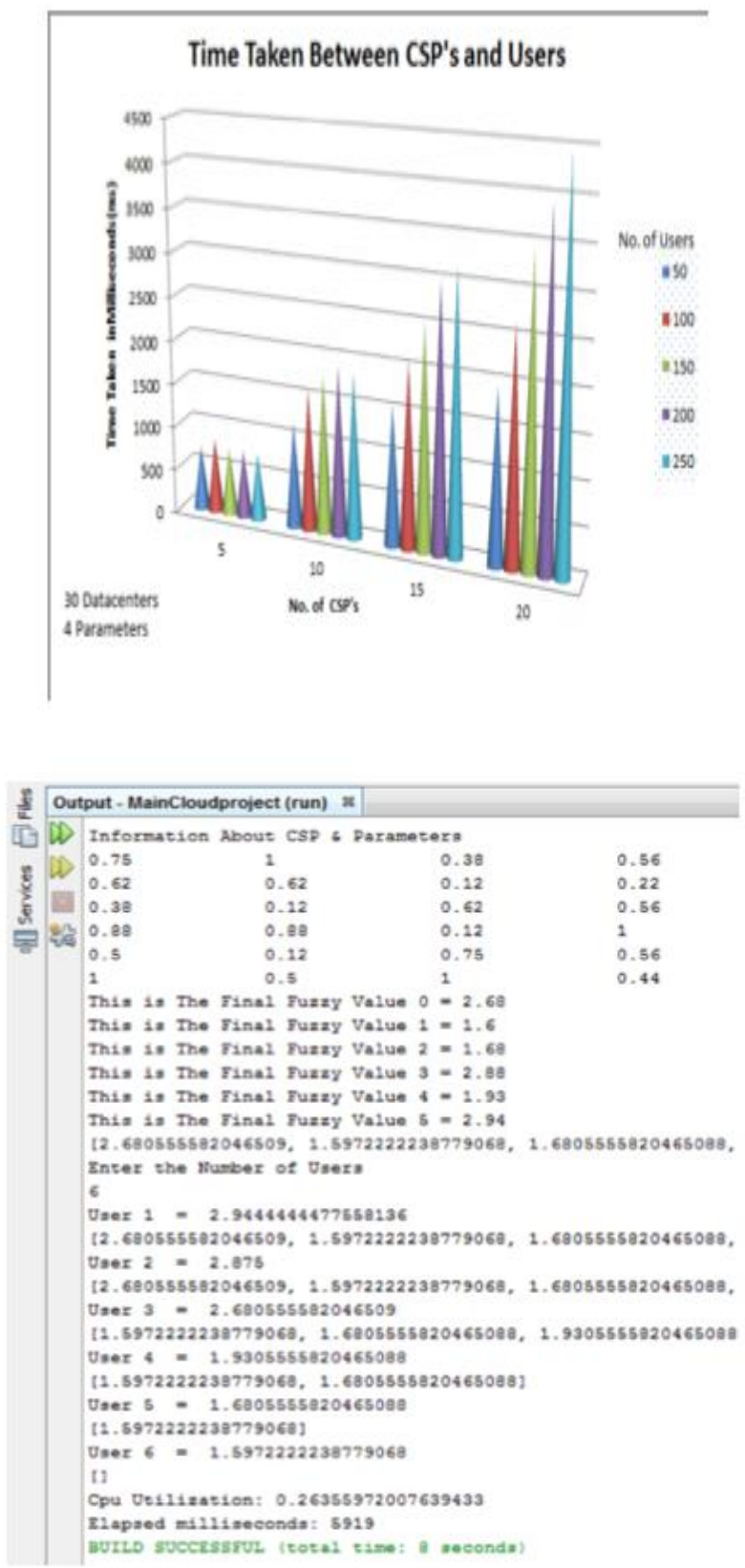

cloud environment in NwtBeans also.

Output 1: Creation of CSP and Datacenters for each CSP

Output 2: Execution of ROSP Algorithm

The graph above represents the time taken by CSP and users in ms. from the above graph we can conclude that:

1. The number of users and CSPs are regularly increasing.

2. The time taken is exponential to the increase of users and CSPs

3. We have generated the results for 30 Datacenters, which can be further expanded.

4. The time taken by the CSP is directly proportional to the parameters.

5. Here we have considered 4 parameters; Virtualization, Application Security, Risk Management and Compliance and Audit.

\section{CONCLUSION \& FUTURE WORK}

In this research work we have proposed the scheduling algorithm for Cloud Broker, which exists between the CSPs and Users. The user/tenant need not to identify the service requirements before submitting their job to the cloud. This is the job of middleware to implement the algorithm and rate CSPs on the basis of their capabilities. All the user/tenant needs to do is to fill the feedback form correctly as to improve the CSPs performance in the future.

Any existing cloud simulators (CloudSim, Grid Sim etc.) can easily implement this model. The scope of this re- search can be extend further, in which apart from taking the cumulative cost function, we can also generate the cost on the basis of individual capability of CSP for an individual attribute. For example if a user needs a CSP whose cost function is maximum for security at- tribute, we can implement the algorithm at each node of the CSP rather than executing it at the broker.

\section{REFERENCES}

[1] J. Octavio, Gutierrez-Garcia, Kwang Mong Sim ,A family

[2] of heuristics for agent-based elastic Cloud bag-of tasks concurrent scheduling, Future Generation Computer Sys-

[3] tems,SciVerseScienceDirect,doi:10.1016/j.future.2012.0 1.005 .

[4] Daji Ergu, Gang Kou, Yi Peng, Yong Shi, Yu Shi ,The

analytic hierarchy process: task scheduling and resource allocation in cloud computing environment, Springer Sci- ence+Business Media, LLC 2011

[5] Jan Komorowski ,Rough Sets: A Tutorial , Department of

[6] Computer and Information Science Norwegian University of Science and Technology (NTNU) 7034 Trondheim, Norway.

[7] Rodrigo N. Calheiros ,CloudSim: A Novel Framework for

[8] Modeling and Simulation of Cloud Computing Infrastructures and Services, Grid Computing and Distributed Systems (GRIDS) Laboratory Department of Computer Science and Software Engineering The University of Melbourne, Australia.

[9] Zdzisaw Pawlak, Rough set theory and its applications, Journal of telecommunication and information technology, vol 3, 2002.

[10] ZHANG Yan-huaa, Feng Leia, Yang Zhia,Optimization of

[11] Cloud Database Route Scheduling Based on Combination of Genetic Algorithm and Ant Colony Algorithm,Procedia Engineering 15 ( 2011 ) 33413345 2011 Published by El- sevier Ltd.

[12] Amit Kumar Sharma ,MADAM ID FOR INTRUSION

[13] DETECTION USING DATA MINING ,IJRIM , Volume 2, Issue 2 (February 2012).

[14] Mehul Mahrishi ,Globally Recorded binary encoded 
[15] Domain Compression algorithm in Column Oriented Databases, Global Journals of Computer Science and technology Vol. 11 issue 23, Page 27-30.

[16] Qi Zhang, Lu Cheng, Raouf Boutaba ,Cloud computing:

[17] state-of-the-art and research challenges,Journal of International Services and Applications, p.7-18, 2010.

[18] Hui Liu ,Ant Colony Optimization Based Service ow

[19] Scheduling with Various QoS Requirements, Cloud Computing in 2011 First ACIS International Symposium on Soft- ware and Network Engineering from School of Computer Science Shanghai University Shanghai City, China.

[20] Ruben Van den Bossche,Cost-Efficient Scheduling Heuristics for Deadline Constrained Workloads on Hybrid Clouds, 2011 Third IEEE International Conference on CloudComputing Technology and Science from Department of Mathematics and Computer Sciences Universiteit Antwerpen Antwerp, Belgium.

[21] T.R.Gopalakrishnan Nair and Vivek Sharma,A Pragmatic Scheduling Approach for Creating Optimal Priority of jobs with Business Values in Cloud Computing,ACC 2012.
[22] Shikharesh Manjumdar,Resource management on cloud

[23] Computing,multifaceted problem and solutions in ACC 2012.

[24] Mehul Mahrishi and Dr.A.Nagaraju ,Optimizing Cloud Service Provider Scheduling by Rough Set model, International Conference on Cloud Computing Techno and Management (ICCEAM-12).

[25] Mehul Mahrishi and Dr.A.Nagaraju, Rating Based For-

[26] mulation for Scheduling of Cloud Service Providers , National Confrance on Emerging treands in IT

[27] Ashish Tiwari, Dr.A.Nagaraju and Mehul Mahrishi, An

[28] Optimized Scheduling Algorithm for Cloud Broker Using Cost Adaptive Modeling, 3rd IEEE (International Advanced Computing Conference-2013).

[29] Z. Pawlak, Rough sets, International Journal of Com-

[30] puter and Information Sciences, 11(5):341356, 1982.

[31] Pawlak (1991), Rough Sets Theoretical Aspects of Rea-

[32] soning about Data, Kluwer Academic Publisher Dordrecht 19. W.Marek Z.Pawlak (1984), Rough Sets and information

[33] Systems, Fundamental Informatica. pp. 105- 115. 